\title{
Poly(vinyl alcohol)/sulfonated Polyester Hydrogels Produced by Freezing and Thawing Technique: Preparation and Characterization
}

\author{
Caio Marcio Paranhos ${ }^{\mathrm{a}}$, Renata Nunes Oliveira $^{\mathrm{a}}$, Bluma Guenther Soares ${ }^{\mathrm{a} *}$, Luiz Antônio Pessan ${ }^{\mathrm{b} *}$ \\ anstituto de Macromoléculas, Universidade Federal do Rio de Janeiro, Centro de Tecnologia, \\ Bloco J, Ilha do Fundão, 21945-970 Rio de Janeiro - RJ, Brazil \\ ${ }^{\mathrm{b}}$ Departamento de Engenharia de Materiais, Universidade Federal de São Carlos, \\ Rodovia Washington Luiz, Km 235, 13565-905 São Carlos - SP, Brazil
}

Received: August 28, 2006; Revised: February 5, 2007

\begin{abstract}
Poly(vinyl alcohol) (PVA) hydrogels were obtained by freezing $\left(-22{ }^{\circ} \mathrm{C}\right)$ and thawing $\left(+25{ }^{\circ} \mathrm{C}\right)$ process, resulting in a semi-interpenetrating polymer network (s-IPN) with a sulfonated polyester (PES). The cryo-gels samples were analysed in terms of its water uptake capacity (WU) as a function of $\mathrm{pH}$, temperature and ionic strength. WU of all samples were relatively high, and they showed a reasonable sensitivity to both $\mathrm{pH}$ and ionic strength. The $\mathrm{pH}$ - and ionic strength dependence is attributed to the dissociation of ionizable groups of PES. The degrees of crystallinity of the cryo-gels, determined by using differential scanning calorimetry (DSC), are lower than pure PVA. Besides, decrease of glass transition temperature $\left(\mathrm{T}_{\mathrm{g}}\right)$ of the samples when compared with pure PVA was observed. This results are due interactions between PVA and PES, which lead to an increase on the amorphous content of the hydrogels. Our results show that, despite of changes in the microstructure of the hydrogels due the presence of the polyelectrolyte, the cryo-gels reveal a long-term stability.
\end{abstract}

Keywords: hydrogels, freezing-thawing process, poly(vinyl alcohol)

\section{Introduction}

Hydrogels can be defined as a three-dimensional network that swell in contact with water or aqueous solutions without dissolve. Poly(vinyl alcohol) (PVA) is a water-soluble polymer, employed in practical applications because of its easy preparation, excellent chemical resistance and physical properties, and because it is completely biodegradable $\mathrm{e}^{1,2}$. However, PVA is a neutral polymer, whose hydrogels have a weak sensitivity to external stimuli, that is, volumetric phase transitions (VPT) are not observed in PVA hydrogels under physiologic conditions. An interesting strategy to reach VPT in PVA hydrogels is the formation of a semi-interpenetrating polymer network (s-IPN) with polyelectrolytes. Polyelectrolytes change their conformation with the degree of dissociation, which is function of quantities such as $\mathrm{pH}$ values, ionic strength and temperature of the external environment solution ${ }^{3-6}$. As a result, the presence of a polyelectrolyte can leads to an overall VPT in the PVA-based hydrogels.

Aqueous solution of PVA can form strong hydrogels through freezing and thawing process. Briefly, PVA solutions are frozen at -5 to $-20{ }^{\circ} \mathrm{C}$ and allowed to thaw to room temperature. This method was firstly reported by $\mathrm{Nambu}^{7,8}$ and it results in the formation of crystallites that serve as physical crosslinks to render the material insoluble in water. This method also was used by Giannouli and Morris to form cryo-gels of xanthan ${ }^{9}$. Several papers have been devoted to evaluate the synthesis and characterization of PVA cryo-gels. Some advantages of these physically crosslinked PVA hydrogels are the non-toxicity, non-carcinogenity and the good biocompatibility. However, with such semicrystalline materials, significant changes in the overall crystalline structure can occurs due aging process, reversible gelation or the presence of a second polymer. To understand these mechanisms and improve the long-term stability of the PVA cryo-gels, Willcox et al. reported that an increase on the number of freezing-thawing cycles applied causes reinforcement on the original network ${ }^{10}$. Lozinsky has

*e-mail: bluma@ima.ufrj.br, pessan@ power.ufscar.br

Article presented at the IV Congresso Latino Americano de Órgãos Artificiais e Biomateriais (COLAOB 2006), August 8 and 11, 2006, Caxambu, MG, Brazil found that the temperature in the vicinity of $-2{ }^{\circ} \mathrm{C}$ can be considered as the thermal condition of the highest efficiency of low molecular mass PVA cryo-gels ${ }^{11}$. On the other hand, Peppas has showed that the higher molecular mass of PVA leads to an undesirable secondary crystallization of network. This fact must be taken into account when hydrogels are designed to pharmaceutical and biological devices, once morphological changes can lead to different swelling degrees and diffusional behavior under physiological stimuli ${ }^{12}$.

In terms of the microstructure, Ricciardi et al. have used several techniques (DSC, ${ }^{1} \mathrm{H}-\mathrm{NMR}$, WAXS and SANS) to confirm a bicontinuous structure of PVA cryo-gels. A PVA-rich phase coexists with a PVA-poor phase, being both phases segregated during the first freezing-thawing cycle. Since the freezing procedure is quite fast, the phase segregation can be not completed and the well-defined network characteristic of PVA cryo-gels is obtained only by repeating the freezing method ${ }^{13-15}$.

The aim of this work was prepare s-IPN hydrogels based on PVA by freezing-thawing technique and evaluates the swelling behavior as a function of $\mathrm{pH}$, temperature and ionic strength. The results are analysed in terms of morphological changes caused by the presence of the polyelectrolyte.

\section{Materials and Methods}

\subsection{Preparation of freezing-thawing hydrogels}

PVA ( $\mathrm{M}_{\mathrm{w}} 120.000$, degree of hydrolysis $99 \%$ ) was supplied by Aldrich. We have used amorphous sulfonated polyester (PES), supplied by Kemira, Belgium ( $\left.\mathrm{M}_{\mathrm{w}} 60.000\right)$, with $20 \% / \mathrm{mol}$ of sulfonated groups. Figure 1 shows the chemical structure of PES. All polymers were used without further purification.

A $10 \%(w / v)$ PVA solution was prepared by adding the solid PVA to the distilled and deionized water into a flask equipped with a reflux 


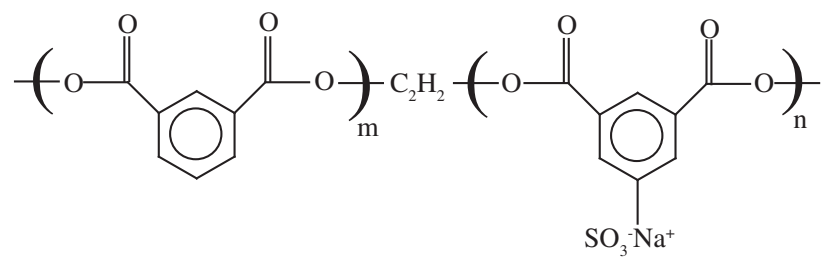

Figure 1. Chemical structure of PES.

condenser. The dissolution of PVA was complete in 4 hours. A $5 \%(\mathrm{w} / \mathrm{v})$ PES solution was prepared by adding the solid PES to distilled and deionized water, and raising the temperature to $60^{\circ} \mathrm{C}$. PVA/PES blends with 90/10, 75/25 and 50/50 (v/v) ratios were prepared. Each blend was dispensed in Petri dishes ( $2.5 \mathrm{~mL} /$ dish). Samples were submitted to five freezing-thawing cycles to obtain hydrogels. With the exception of the first one, each cycle involved lowering the temperature to $-22{ }^{\circ} \mathrm{C}$, standing at this temperature for 1 hour, then raising the temperature to room temperature, standing at this temperature for 30 minutes. The first cycle differed from the others because of a longer standing time at $-22{ }^{\circ} \mathrm{C}(17$ hours $)$. The obtained hydrogels were dried in a dissecator under vacuum until show constant weight.

\subsection{Swelling behavior}

The water uptake capacity (WU) was measured in various buffer solutions. Preweighed dry samples were immersed in solutions with various $\mathrm{pH}$, temperature and ionic strength until they swelled to equilibrium. It was confirmed that 24 hours equilibration was enough to reach the equilibrium swelling of the samples. After surface water was removed with filter paper, the fully swollen samples were weighed. WU was calculated from the following equation,

$$
\mathrm{WU}=\left(\frac{\mathrm{W}_{\mathrm{s}}-\mathrm{W}_{\mathrm{d}}}{\mathrm{W}_{\mathrm{d}}}\right) \times 100
$$

where $\mathrm{w}_{\mathrm{s}}$ is the weight of the swollen sample at the equilibrium and $\mathrm{w}_{\mathrm{d}}$ is the weight of the dry sample. The experiments were done in triplicate.

\subsection{Thermal analysis}

Differential scanning calorimetry (DSC-7, Perkin-Elmer) was used to evaluate the degree of crystallinity of the samples. In a typical procedure, 5-10 $\mathrm{mg}$ of a dried sample was placed in an aluminum pan and heated at a scanning rate of $10{ }^{\circ} \mathrm{C} / \mathrm{min}$ from 40 to $250{ }^{\circ} \mathrm{C}$ under a nitrogen purge.

Dynamic-mechanical analysis (DMA Q-800, TA Instruments) was performed to evaluate the glass transition temperature $\left(\mathrm{T}_{\mathrm{g}}\right)$ of the cryo-gels samples. It was used a tensile mode apparatus at constant strain. Tg of the samples were reported as the maximum tan delta peak over a temperature range from -20 to $120^{\circ} \mathrm{C}$ at a heating rate of $3{ }^{\circ} \mathrm{C} / \mathrm{min}$ and frequency of $1 \mathrm{~Hz}$.

\subsection{Long-term stability of the hydrogels}

The PVA/PES hydrogels samples were immersed in distilled water at room temperature during several periods of time until 15 days. Aliquots of $10 \mathrm{~mL}$ from the immersing solution were withdrawn and treated with $0.65 \mathrm{M}$ boric acid and $0.05 \mathrm{M} \mathrm{I}_{2} / 0.15 \mathrm{KI}$ solutions at $25{ }^{\circ} \mathrm{C}$. The PVA chains that do not taken place on the hydrogel formation readily form a green complex with iodine ions in the presence of boric acid ${ }^{16}$. Visible light absorbance was measured at $670 \mathrm{~nm}$ in a Cary 100 (Varian) spectrophotometer.

\section{Results and Discussion}

Figure 2 shows the water uptake capacity of various samples as a function of $\mathrm{pH}$. It is clear that the s-IPN hydrogels are more labile near

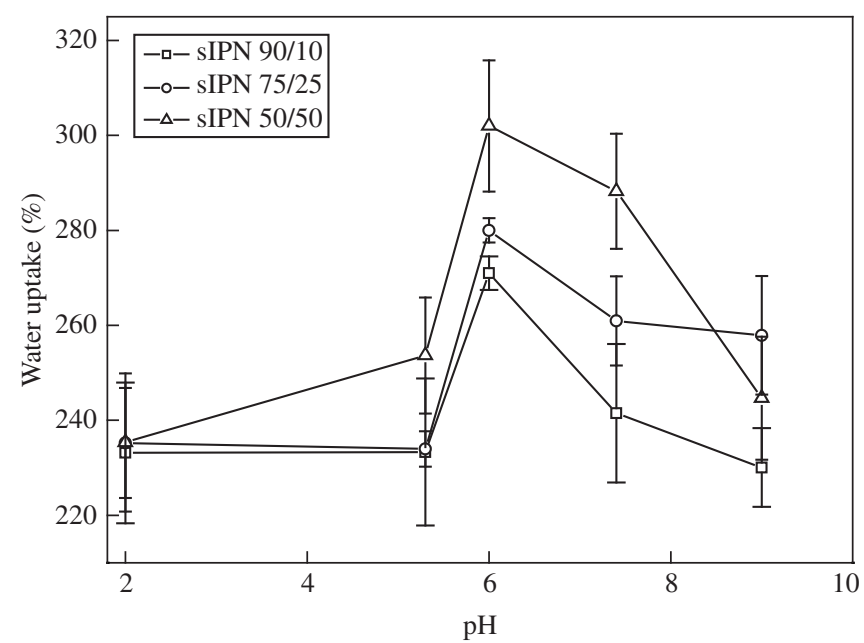

Figure 2. Equilibrium water uptake (WU) of the s-IPN PVA/PES hydrogels in various $\mathrm{pH}$ buffer solutions at $25^{\circ} \mathrm{C}$.

the neutrality. The water uptake capacity of s-IPN hydrogels increases when the $\mathrm{pH}$ of the buffer is above $\mathrm{pH} 4$, which is caused by the dissociation of ionic bonds in the s-IPN hydrogels. Dissociation of the sulfonated group of the polyelectrolyte occurs, causing an increase in the osmotic pressure due the counter-ion mobility into the hydrogels. Under extremely low $\mathrm{pH}$ conditions, most of the PES sulfonated groups are part of $-\mathrm{SO}_{3} \mathrm{H}$. On the other hand at high $\mathrm{pH}$ values, most sulfonated groups of PES are $-\mathrm{SO}_{3}$ - forms. As a result, intermolecular hydrogen bonds between PVA and PES are diminished, contributing to an overall improvement of the degree of swelling of the hydrogels.

However, a decrease in the water uptake capacity can be seen to higher $\mathrm{pH}$. It is due the presence of $\mathrm{OH}^{-}$groups that tend to shield the sulfonated groups of PES, reducing the repulsion between the fixed charges on the polymeric chains, thus limiting the hydration of the s-IPN hydrogels.

Temperature-dependant equilibrium swelling behavior of s-IPN hydrogels in distilled water at a temperature range from 25 to $45^{\circ} \mathrm{C}$ is shown in Figure 3. It can be seen that all PVA/PES samples show a slight temperature-responsive behavior, revealing an increase on the water uptake when compared with pure PVA.

This behavior can be attributed to the thermal expansion of the PVA matrix. The thermal mobility of the chains with temperature contributes directly on the dissociation of the inter- and intramolecular hydrogen bonding between PVA and PES. However, it seems that the thermal mobility of PES has an important role on temperatureresponsive behavior, since the glass transition temperature of PES $\left(\sim 45^{\circ} \mathrm{C}\right)$ is situated on the vicinity of the experimental range.

The effect of the ionic strength of the aqueous solution on the swelling behavior of s-IPN hydrogels was also studied. As shown in Figure 4, the WU of the hydrogels decreases with increasing ionic strength for all the samples. With the content of the PES increasing, WU increases, indicating the contribution of PES chains.

Due the presence of small electrolytes, the affinity action between the sulfonated groups of PES with the water is destroyed. It causes the collapse of the network and the decrease the WU. When the content of small electrolytes is lower, the effect of the ionic strength on the swelling of s-IPN hydrogels is remarkable. In the case of 50/50 hydrogel sample, the WU decreases $150 \%$ when the ionic strength increases from $10^{-4}$ to 1 .

The effect of PES content on $\mathrm{T}_{\mathrm{m}}$ and $\Delta \mathrm{H}_{\mathrm{m}}$ of the s-IPN hydrogels was investigated. As can be seen in Figure 5 that there are no signifi- 
cant changes on $\mathrm{T}_{\mathrm{m}}$ with the PES content. This result indicates that the presence of PES chains does not lead to a decrease on the overall crystallite lamellar size of the PVA.

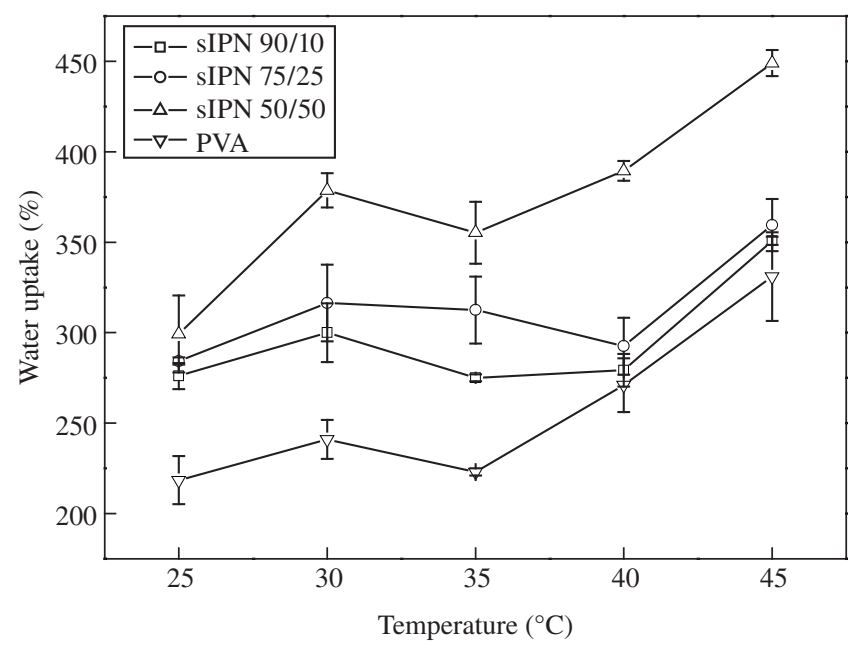

Figure 3. Equilibrium water uptake (WU) of the s-IPN PVA/PES hydrogels as a function of temperature.

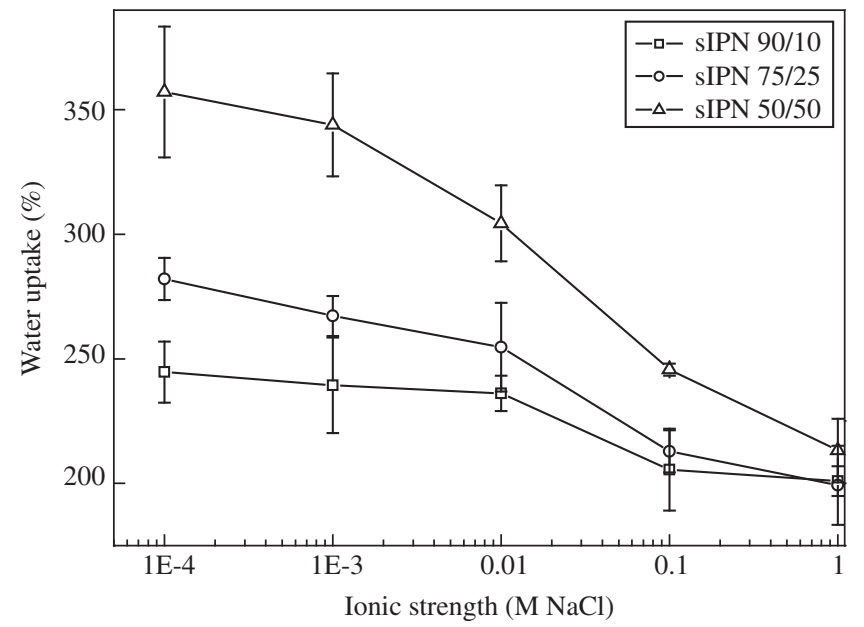

Figure 4. Effect of the ionic strength of the solution on the water uptake (WU) of the s-IPN PVA/PES hydrogels.

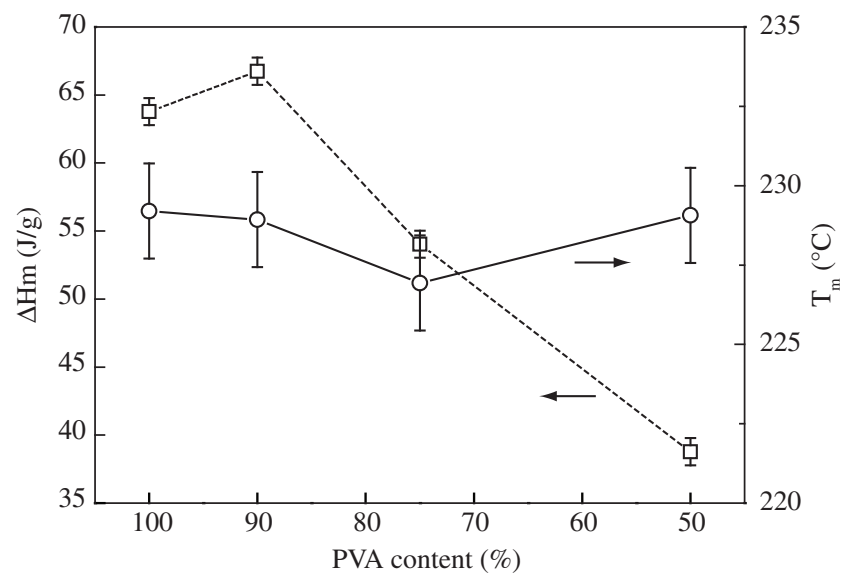

Figure 5. Effect of the PES content on the $\mathrm{T}_{\mathrm{m}}$ and $\Delta \mathrm{H}_{\mathrm{m}}$ of the s-IPN PVA/PES hydrogels.
However, the presence of PES causes a decrease on $\Delta \mathrm{H}_{\mathrm{m}}$ for the s-IPN hydrogels. Therefore, the degree of crystallinity of the PVA is perturbed by the presence of PES chains, that is, the amorphous fraction of PVA increases with the increase of the PES content. The PVA glass transition temperature $\left(\mathrm{T}_{\mathrm{g}}\right)$ shifts towards lower values, due the presence of PES, as can be seen in Figure 6. In addition, the blends are transparent, suggesting no phase separation under macroscopic scale.

This result confirms a strong interaction between PVA and PES. Since PVA chains available to interact with PES are mainly situated in the amorphous fraction of PVA, the degree of crystallinity decreases with PES content. Recently, we have reported a microstructural analysis of the PVA/PES cryo-gel, using the technique based on positron annihilation lifetime spectroscopy (PALS). It was observed an increase of the average free volume of the samples with increasing the PES content ${ }^{17}$, confirming the interactions between the blend components and also the role of amorphous fraction of cryo-gels samples on the interactions mechanisms between PVA and PES. Since the crystallinity is responsible for the crosslinking points in this system, a decrease of this parameter also decreases the number of available crosslinking points, leading to an increase on the water uptake capacity of the hydrogels, as discussed.

The stability experiments for the s-IPN hydrogels shown a maximum PVA dissolution content of $1.2 \%$ for all the samples after 15 days. It seems that the PES chains do not promote a disordering process of the PVA crystalline domains, assuring the long-term stability of the PVA/ PES hydrogels ${ }^{18}$. This characteristic of the PVA/PES cryo-gels can be considered during the design and manufacturing of pharmaceutical and biomedical devices for controlled delivery of bioactive substances.

\section{Conclusions}

The presence of a polyelectrolyte on the formation of a semiinterpenetrating polymer network PVA hydrogels through freezing and thawing process results in an environment-sensitivity system. Volumetric phase transition was reached in PVA/PES cryo-gels as a function of $\mathrm{pH}$ and ionic strength. Polymer-polymer interactions and the ionic nature of the polyelectrolyte play an important role on the obtained results. Besides, the perturbation of the crystalline domains of PVA as a function of the PES content leads to an increase on the water uptake capacity of the hydrogels. However, these structural changes seem not contribute to collapse the system along the time, remaining their original shape and swelling behavior. These hydro-

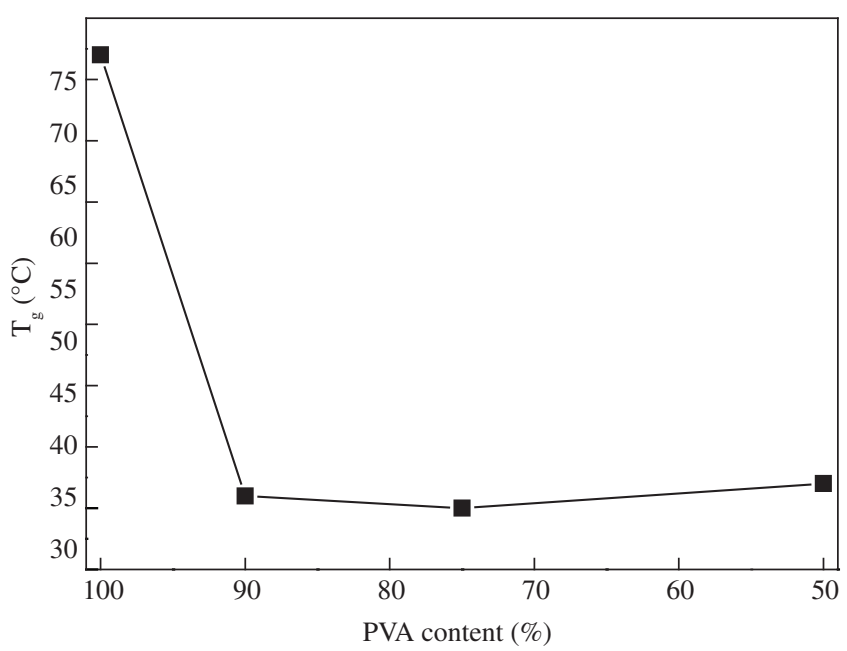

Figure 6. PVA glass transition temperature as a function of PES content 
gels therefore can be an interesting alternative devices to controlled release of bioactive agents, whose stability and swelling capacity are the most important factors to a successful applications.

\section{Acknowledgments}

This work was supported by Coordenação de Aperfeiçoamento de Pessoal de Nível Superior (CAPES), Conselho Nacional do Desenvolvimento Científico e Tecnológico (CNPq) and Fundação de Amparo à Pesquisa do Estado do Rio de Janeiro (FAPERJ).

\section{References}

1. Finch CA, editor, Polyvinyl alcohol, properties and applications. New York: John Wiley; 1973.

2. Peppas NA, editor, Hydrogels in medicine and phamarcy. Vol 1. Boca Raton: CRC Press; 1986.

3. Byun J, Lee YM, Cho C. Swelling of thermosensitive interpenetrating polymer networks composed of poly(vinyl alcohol) and poly(acrylic acid). Journal of Applied Polymer Science. 1996; 61(4):697-702.

4. Kim SJ, Park SJ, Kim SI. Swelling behavior of interpenetrating polymer network hydrogels composed of poly(vinyl alcohol) and chitosan. Reactive and Functional Polymers. 2003; 55(1):53-59.

5. El-Zawawy WK. Preparation of hydrogel from green polymer. Polymers for Advanced Technologies. 2005; 16(1):48-54.

6. Mucha M, Ludwiczack S, Kawinska M. Kinetics of water sorption by chitosan and its blends with poly(vinyl alcohol). Carbohydrate Polymers. 2005; 62(1):42-49.

7. Nambu M, inventor. Nippon Oil Co., assignee. Method of preparing polyvinyl alcohol gel. Japanese patent 130543; 1982.

8. Tamura K, Ike O, Hitomi S, Isobe J, Shimizu Y, Namu M. A New Hydeogel and Its Medical Application. Transactions - American Society for Artificial Internal Organs. 1986; 32(1):605-608.
9. Giannouli P, Morris ER. Cryogelation of xanthan. Food Hydrocolloids 2003; 17(4):495-501.

10. Willcox PJ, Howie Jr DW, Schmidt-Rohr K, Hoagland DA, Gido SP, Pudjianto $\mathrm{S}$ et al. Microstructure of poly(vinyl alcohol) hydrogels produced by freeze/thaw cycling. Journal of Polymer Science: Part B: Polymer Physics. 1999; 37(24):3438-3454.

11. Lozinsky VI, Damshkaln LG. Study of cryostructuration of polymer systems. XVII. Poly(vinyl alcohol) cryogels: Dynamics of the cryotropic gel formation. Journal of Applied Polymer Science. 2000; 77(9):2017-2023.

12. Hassan CM, Ward JH, Peppas NA. Modeling of crystal dissolution of poly(vinyl alcohol) gels produced by freezing/thawing processes. Polymer. 2000; 41(18):6729-6739.

13. Ricciardi R, Auriemma F, De Rosa C, Lauprête F. X-ray diffraction analysis of poly(vinyl alcohol) hydrogels, obtained by freezing and thawing techniques. Macromolecules. 2004; 37(5):1921-1927.

14. Ricciardi R, Auriemma F, Gaillet C, De Rosa C, Lauprête F. Investigation of the crystallinity of freeze/thaw poly(vinyl alcohol) hydrogels by different techniques. Macromolecules 2004; 37(25):9510-9516.

15. Ricciardi R, Mangiapia G, Lo Celso F, Paduano L, Triolo R, Auriemma F et al. Structural organization of poly(vinyl alcohol) hydrogels obtained by freezing and thawing techniques: A SANS study. Chemistry of Materials. 2005; 17(5):1183-1189.

16. Joshi DP, Lan-Chun-Fung YL, Pritchard JW. Determination of polyvinylalcohol via its complex with boric-acid and iodine. Analytica Chimica Acta. 1979; 104(1):153-160.

17. Paranhos CM, Oliveira RN, Soares BG, Pessan LA, Freitas DS, Windmöller D et al. Microstructural evaluation of poly(vinyl alcohol)-based hydrogels obtained by freezing-thawing technique: thermal analysis and positron annihilation. Journal of Applied Polymer Science. in press.

18. Hassan CM, Peppas NA. Structure and morphology of freeze/thawed PVA hydrogels. Macromolecules. 2000; 33(7):2472-2479. 